\title{
Schwangere können Krebstherapie früh starten
}

\author{
Werdende Mütter erhalten zum Schutz des Kindes nur ein Minimum an Therapien. Wird ein neues \\ Karzinom entdeckt, kann die Chemo- oder Radiotherapie aber nach dem 1. Trimester beginnen.
}

_ In einer multizentrischen Fall-Kontroll-Studie wurden 129 Kinder beobachtet, bei deren Müttern während der Schwangerschaft erstmals ein Karzinom diagnostiziert wurde. Nach dem 1. Trimester erhielten 74,4\% der Schwangeren eine Chemotherapie und 8,5\% eine Radiotherapie. 10,1\% wurden operiert, $1,6 \%$ erhielten andere Medikamente, 10,9\% blieben ohne spezifische Therapie. Die Kinder wurden nach der Geburt im Mittel 22 Monate lang pädiatrisch, neurologisch, kardial und kognitiv untersucht. Als Kontrolle dienten Kinder gesunder Mütter, die im gleichen Gestationsalter entbunden wurden.

Bei den Nachuntersuchungen wurden im Vergleich zur Kontrollgruppe keine signifikanten pathologischen Befunde erhoben: Geburtsgewicht und kognitive Entwicklung waren normal. Kardiale Veränderungen konnten per EKG und Echokardiografie ausgeschlossen werden - auch bei Kindern von Müttern mit Anthrazyklintherapie.

- Amant F et al. for the International Network on Cancer, In-
fertility, and Pregnancy (INCIP): Pediatric Outcome after Ma-
ternal Cancer Diagnosed during Pregnancy. N Engl IMed.
2015;373:1824-34
KOM MENTAR
Bei der Verordnung von Medikamenten in
der Schwangerschaft wird größte Zurückhal-
tung empfohlen und geübt, und das nicht
nur bei Substanzen mit bekannter embryo-
toxischer und teratogener Wirkung. Bei strik-
ter Anwendung müssten deshalb Chemo-

- Amant $F$ et al. for the International Network on Cancer, Infertility, and Pregnancy (INCIP): Pediatric Outcome after Maternal Cancer Diagnosed during Pregnancy. N Engl J Med. und Radiotherapie in der Schwangerschaft teils um Monate zurückgestellt werden. Eine onkologische Therapie wird erst nach der vulnerablen Phase der Organogenese begonnen, also erst ab dem 2. Trimester. In der vorliegenden und auch in anderen Studien zeigte sich unter diesen Voraussetzungen keine Zunahme von Missbildungen. Die Erfahrungen mit der Therapie von Malignomen in der Schwangerschaft bleiben auch nach diesem Bericht begrenzt. Doch die Ergebnisse stimmen zuversichtlich. Sie könnten dazu beitragen, Schwangerschaftsabbrüche noch intensiver zu hinterfragen. Auch geben sie Sicherheit, wenn eine onkologische Therapie zeitgerecht, also ohne unnötige Verzögerungen eingeleitet werden soll.

Prof. Dr. med. H. Holzgreve

\section{Am ganzen Körper finden sich Teleangiektasien}

Eine 58-jährige Frau berichtete über intermittierende rektale Blutabgänge in den vergangenen sechs Wochen. Sie litt unter zunehmender Müdigkeit und Atemnot. Aus der früheren

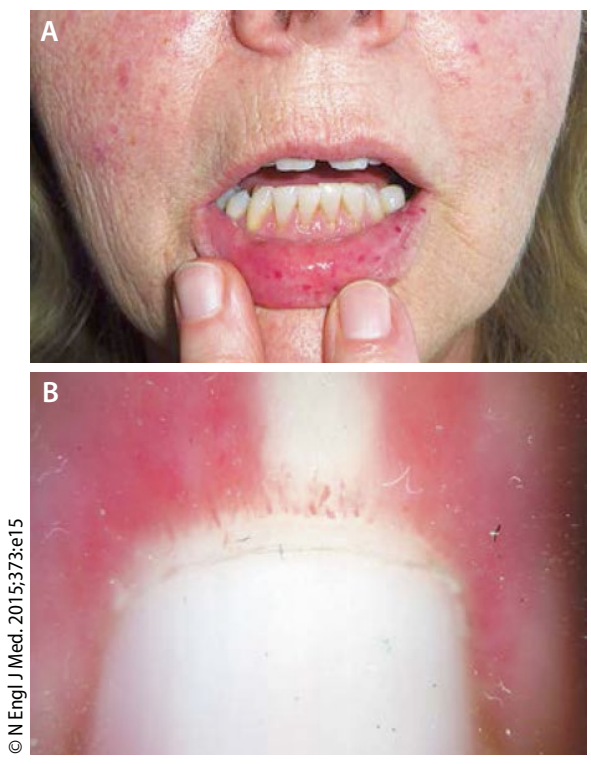

Anamnese waren wiederholte Episoden von spontanem Nasenbluten bekannt, die nie richtig abgeklärt worden waren. Bei der körperlichen Untersuchung zeigten sich Teleangiekta-

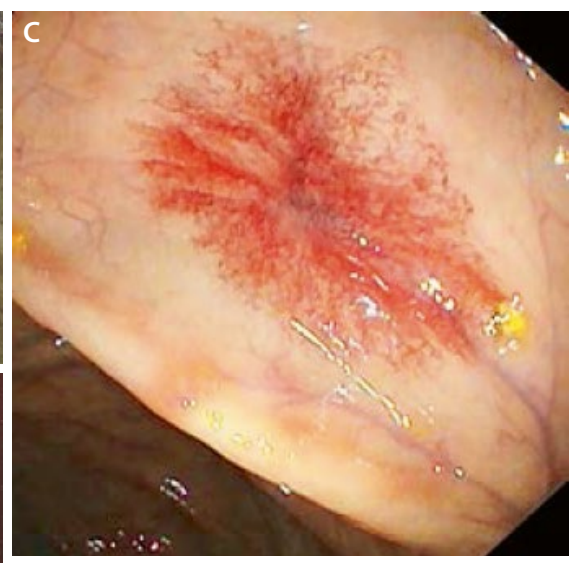

Teleangiektasien an der Lippenschleimhaut (A), am Nagelfalz (B) und im Dickdarm (C). sien an der Lippenschleimhaut (Abb. A) und am Nagelfalz (Abb. B). Im Gespräch stellte sich heraus, dass auch die Mutter häufig Nasenbluten hatte und bei ihr ähnliche Hautveränderungen bestanden. Im Labor zeigte sich eine ausgeprägte Eisenmangelanämie mit einem $\mathrm{Hb}$ von $8,1 \mathrm{~g} / \mathrm{dl}$ und einem mittleren korpuskulären Volumen (MCV) von $72 \mathrm{fl}$. In der Koloskopie stellte mal zahlreiche arteriovenöse Malformationen und Teleangiektasien fest (Abb. C). Pulmonale oder zerebrale Gefäßmalformationen fanden sich im Zuge der weiteren Abklärung nicht.

Anhand der klinischen Befunde stellte man die Diagnose einer hereditären hämorrhagischen Teleangiektasie, die auch als Osler-WeberRendu-Syndrom bekannt ist. Eine molekularbiologische Analyse ergab eine Genmutation im Endoglin-Gen bei der Patientin und ihrer Mutter. Damit war die molekulare Diagnose gesichert.

Prof. Dr. med. H. S. FüeßI

- Pérez-Belmonte LM, Gomez-MoyanoE

(luismiguelpb@hotmail.com). Osler-Weber-Rendu-Syndrome. NEngl J Med. 2015;373:e15 